\title{
Behavior of Quantum Fisher Information of Bell Pairs under Decoherence Channels
}

\author{
F. Ozaydin ${ }^{a}$, A.A. Altintas ${ }^{b}$, S. Bugu $^{c}$ And C. Yesilyurt $^{b}$ \\ ${ }^{a}$ Isik University, Istanbul, Turkey \\ ${ }^{b}$ Okan University, Istanbul, Turkey \\ ${ }^{c}$ Istanbul University, Istanbul, Turkey
}

\begin{abstract}
Quantum Fisher information has recently been an essential tool for analyzing the phase sensitivity of the quantum states in various quantum tasks, requiring high precision, such as quantum clock synchronization, positioning and many applications which include quantum interferometers. Due to the interactions with the environment, all quantum systems are subject to various decoherence effects. Therefore the research on quantum Fisher information under decoherence has been recently attracting more attention. In this work, analyzing the quantum Fisher information, we study the phase sensitivity of bipartite quantum correlations, in particular four Bell pairs amplitude damping channels. For a specific Bell state we arrive at similar results of Greenberger-Horne-Zeilinger (GHZ) states (as expected). For the other three Bell states, we present our results which point the interesting behavior of quantum Fisher information with respect to the decoherence rate. We also find the regions where the quantum Fisher information exhibits discontinuities.
\end{abstract}

DOI: $10.12693 /$ APhysPolA.125.606

PACS: 03.67.-a, 03.65.Ud, 03.65.Yz

\section{Introduction}

Quantum Fisher information (QFI), extending the classical Fisher information [1, 2], has recently attracted more attention due to its importance for analyzing the usefulness of quantum states for the tasks requiring phase sensitivity. In particular, the higher QFI is, the higher precision can be achieved in such tasks, for example in clock synchronization [3] and in quantum frequency standards [4]. Although some pure entangled states can surpass the classical limit, this is not the case for all the entangled states [5]. The unavoidable interactions between the quantum system and the environment, not only degrades the entanglement but also decreases the QFI of the system. Therefore studying the QFI of the quantum systems, especially under decoherence, is a key step for quantum technologies. Recently, a single parameter, $\chi^{2}$, has been introduced for the phase sensitivity that an arbitrary (possibly mixed) quantum system can provide, which depends only on the system itself [6]. For a general quantum system, the condition $\chi^{2}<1$ is sufficient to imply that the system is multipartite entangled and can provide a better phase sensitivity than any separable system. Such quantum systems are therefore called "useful". For two-level $\mathrm{N}$-particle quantum systems, the Cramer-Rao bound (QCB) [1,2] is given by

$$
\Delta \phi_{\mathrm{QCB}} \equiv \frac{1}{\sqrt{N_{m} F}}
$$

where $N_{m}$ is the number of experiments that could be performed over the system in a task including interferometers and $F$ is the quantum Fisher information. Introducing the fictitious angular momentum operators $J_{n}$ in the $\boldsymbol{n}$-th direction of the normalized three-dimensional vector, in terms of the Pauli matrices $\sigma$,

$$
J_{\boldsymbol{n}}=\sum_{\alpha=x, y, z} \frac{1}{2} n_{\alpha} \sigma_{\alpha}
$$

the Fisher information of a quantum state $\rho$ for $J_{\boldsymbol{n}}$ is given in [7] in terms of a symmetric matrix $C$ :

$$
F\left(\rho, J_{\boldsymbol{n}}\right)=\sum_{i \neq j} \frac{2\left(p_{i}-p_{j}\right)^{2}}{p_{i}+p_{j}}\left|\left\langle i\left|J_{\boldsymbol{n}}\right| j\right\rangle\right|^{2}=\boldsymbol{n} C \boldsymbol{n}^{\mathrm{T}},
$$

where each $p_{i}$ and $|i\rangle$ are the eigenvalues and the eigenvectors of $\rho$, respectively, and the matrix elements of $C$ are given as

$$
\begin{aligned}
C_{k l} & =\sum_{i \neq j} \frac{\left(p_{i}-p_{j}\right)^{2}}{p_{i}+p_{j}} \\
& \times\left[\left\langle i\left|J_{k}\right| j\right\rangle\left\langle j\left|J_{l}\right| i\right\rangle+\left\langle i\left|J_{l}\right| j\right\rangle\left\langle j\left|J_{k}\right| i\right\rangle\right] .
\end{aligned}
$$

Choosing the largest $F$ among $\boldsymbol{n}$, and averaging over the number of particles $N$, the Fisher information is given by the largest eigenvalue of the $C$ matrix, i.e.

$$
\bar{F}_{\max }=\frac{1}{N} \max _{\boldsymbol{n}} F\left(\rho, J_{\boldsymbol{n}}\right)=\frac{\lambda_{\max }}{N} .
$$

For $N_{m}=1$, the best separable states of $N$ particles can achieve $\bar{F}_{\max }=N$ and the best multipartite entangled state, actually a GHZ state can achieve $\bar{F}_{\max }=$ $N^{2}$. From the view point of the phase sensitivity, the limit of the separable states is called shot noise limit, $\Delta \phi_{\mathrm{SN}}$ and the fundamental limit is called the Heisenberg limit, $\Delta \phi_{\mathrm{HL}}$. It is also shown that uncertainty principle allows surpassing the fundamental limit [8]. The pure multipartite entangled states which surpass the shot-noise limit is an interesting research direction; since GHZ states achieve the fundamental limit but $W$ states cannot achieve even the shot-noise limit, we have recently studied the superposition of these two main multipartite entangled states and have shown the dependence of the Fisher information to the superposition coefficient [9]. 
The quantum Fisher information of systems under decoherence is also an important direction and GHZ states has been studied for three main decoherence channels, i.e. amplitude damping, phase damping, and depolarizing and the behavior of quantum Fisher information has been presented with the interesting sudden change points [7].

\section{QFI of Bell states under amplitude damping noise}

In this work, we study the Bell states under amplitude damping noise and report discontinuity in the quantum Fisher information of the states. We present our results in Fig.1. With the standard definition, the four Bell states are

$$
\begin{aligned}
& \left|\Psi_{a}\right\rangle=\frac{|00\rangle+|11\rangle}{\sqrt{2}}, \quad\left|\Psi_{b}\right\rangle=\frac{|00\rangle-|11\rangle}{\sqrt{2}}, \\
& \left|\Psi_{c}\right\rangle=\frac{|01\rangle+|10\rangle}{\sqrt{2}}, \quad\left|\Psi_{d}\right\rangle=\frac{|01\rangle-|10\rangle}{\sqrt{2}},
\end{aligned}
$$

and the Kraus operators of the amplitude damping channel,

$$
E_{0}=|0\rangle\langle 0|+\sqrt{1-p}| 1\rangle\left\langle 1\left|, \quad E_{1}=\sqrt{p}\right| 0\right\rangle\langle 1| .
$$

Note that GHZ state is an $N$-particle extension of $\left|\Psi_{a}\right\rangle$ and $W$ state can be considered as an $N$ particle extension of $\left|\Psi_{c}\right\rangle$. Therefore applying the amplitude damping noise to the states, for $\left|\Psi_{a}\right\rangle$ we obviously reproduce the result of [7] for $N=2$ and $\left|\Psi_{c}\right\rangle$ can be considered as a step for studying the quantum Fisher information of $W$ states under decoherence.

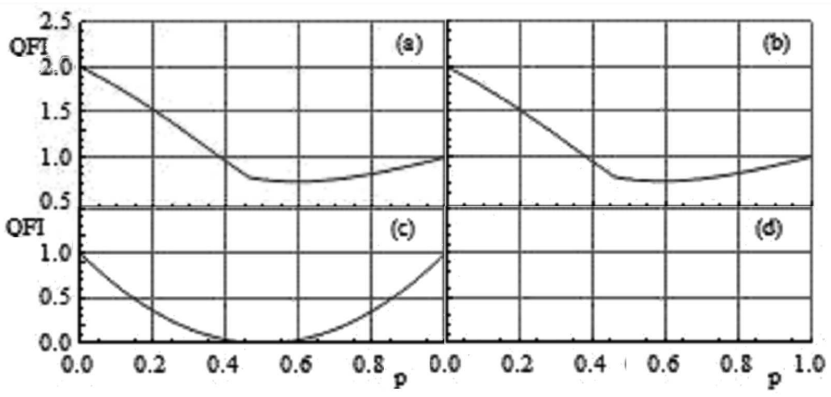

Fig. 1. Quantum Fisher information of Bell states under amplitude damping noise. For the first three states, when there is no decoherence, QFI $=2$ but for the fourth state QFI $=0$ even when the state is pure. (a) Bipartite case of [7]. (b) The relative phase has no effect when compared to the first state. (c) Sharp decrease in QFI: when the third state is pure, QFI $=2$ but even a very low rate of decoherence decreases the QFI to the level of a pure separable state of two particles. (d) Fisher information of fourth Bell state is always zero, no matter the state is pure or subject to an amplitude damping decoherence of any rate.

When $\left|\Psi_{c}\right\rangle$ is subjected to even a small rate of decoherence, we observe a discontinuity in the Fisher informa- tion: a sharp decrease from 2 to 1 , which is the shot-noise limit. As the decoherence rate increases, QFI decreases below the shot-noise limit and when the state is completely decohered, the QFI reaches the shot-noise limit. Another interesting finding is that, although QFI of $\left|\Psi_{b}\right\rangle$ behaves the same as $\left|\Psi_{a}\right\rangle$, this is not the case for the $\left|\Psi_{d}\right\rangle$ and $\left|\Psi_{c}\right\rangle$, i.e. the relative phase of the superposition has a killing effect on QFI, even without decoherence.

The Bell states can be converted to each other by local operations and classical communications (LOCC), but this is not the case for multipartite entangled states, such as GHZ and $W$ states [10]. Although pure $W$ states cannot achieve even the shot-noise limit, there may be some quantum tasks requiring both phase sensitivity and $W$ states in particular. Therefore studying the QFI of $W$ states of $N$ particles under decoherence can be considered as a future study, which we studied herein for the specific case for $N=2$ (as the third Bell state). Another interesting question is that whether there is a noise that increases the QFI of a given quantum state.

Since optical setups for preparing large-scale polarization entangled photonic states, in particular $\mathrm{W}$ states is recently proposed for the ideal case [11-13], studying the QFI of these states during the preparation process in the non-ideal case where photon losses and imperfections of the experimental setup are taken into account may help bringing these proposals into physical implementation with current photonics technology.

\section{References}

[1] C.W. Helstrom, Quantum Detection and Estimation Theory, Academic Press, New York 1976.

[2] A.S. Holevo, Probabilistic and Statistical Aspects of Quantum Theory, North-Holland, Amsterdam 1982.

[3] R. Jozsa, D.S. Abrams, J.P. Dowling, C.P. Williams, Phys. Rev. Lett. 85, 2010 (2000).

[4] J.J. Bollinger, W.M. Itano, D.J. Wineland, D.J. Heinzen, Phys. Rev. A 54, R4649 (1996).

[5] P. Hyllus, O. Gühne, A. Smerzi, Phys. Rev. A 82, 012337 (2010).

[6] L. Pezze, A. Smerzi, Phys. Rev. Lett. 102, 100401 (2009).

[7] J. Ma, Y.-X. Huang, X. Wang, C.P. Sun, Phys. Rev. A 84, 022302 (2011).

[8] V. Giovannetti, S. Lloyd, L. Maccone, Science 306, 5700 (2004).

[9] F. Ozaydin, A.A. Altintas, S. Bugu, C. Yesilyurt, Int. J. Theor. Phys. 52, 2977 (2013).

[10] W. Dur, G. Vidal, J.I. Cirac, Phys. Rev. A 62, 062314 (2000).

[11] S. K. Ozdemir, E. Matsunaga, T. Tashima, T. Yamamoto, M. Koashi, N. Imoto, New J. Phys. 13, 103003 (2011).

[12] S. Bugu, C. Yesilyurt, F. Ozaydin, Phys. Rev. A 87, 032331 (2013).

[13] C. Yesilyurt, S. Bugu, F. Ozaydin, Quantum Inf. Process 12, 2965 (2013). 